\title{
"Y ahora cómo lo digo", la asignatura pendiente en educación superior
}

\section{M'Pilar Ribate $^{\mathrm{a}}$, Clara Llanas ${ }^{\mathrm{b}}$, Laura Lomba ${ }^{\mathrm{b}}$, Estefanía Zuriaga ${ }^{\mathrm{c}}$, Víctor López ${ }^{\mathrm{d}}$ Beatriz Ginere ${ }^{e}$, Cristina B. García .}

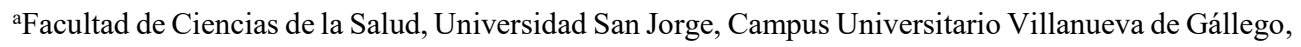

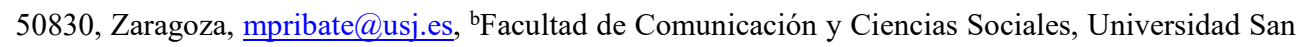

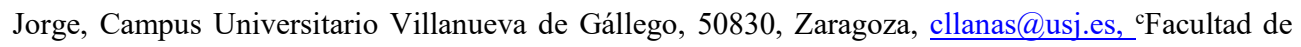
Ciencias de la Salud, Universidad San Jorge, Campus Universitario Villanueva de Gállego, 50830, Zaragoza, 1lomba@usj.es, ${ }^{\mathrm{d}}$ Facultad de Ciencias de la Salud, Universidad San Jorge, Campus

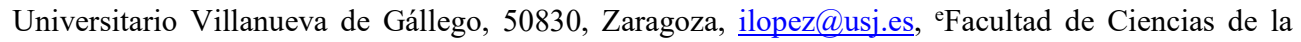
Salud, Universidad San Jorge, Campus Universitario Villanueva de Gállego, 50830, Zaragoza, bginer@usj.es, ${ }^{\text {fF}}$ Facultad de Ciencias de la Salud, Universidad San Jorge, Campus Universitario Villanueva de Gállego, 50830, Zaragoza, cbgarcia@usj.es.

\begin{abstract}
An inclusive society assures that all people have the same opportunities and resources necessary to participate fully in economic, social, political and cultural life. A vulnerable person is who has a weakness (family, relational, socio-economic ...) that makes it at risk of social exclusion. People with intellectual disabilities are a group that would be included in this population at risk reducing their participation in social life, increasing their insecurity and low self-esteem. The education projects focused on values allow to generate positive expectations in these groups and these people can develop in a more autonomous environment. The Learning-Service activities are a tool to acquire learning through experience because students must plan, develop and evaluate the activities aimed at improving the community.

With these experiences the students of different courses of the Degree in Pharmacy and Infant and Primary Education participated in different activities to improve communication skills between people with intellectual disabilities and health professionals. Likewise, the students were able to deepen more in the contents of the subjects involved and put into practice their communication skills, as well as becoming aware of the problems that surround this population.
\end{abstract}


Keywords: inclusión, people with intellectual disabilities, Learning-Service, comunication skills, health professionals.

\begin{abstract}
Resumen
Una sociedad inclusiva asegura a todas las personas las oportunidades y recursos necesarios para participar plenamente en la vida económica, social, política y cultural. Una persona vulnerable es aquella que padece una debilidad (familiar, relacional, socio-económica ...) que hace que se encuentre en una situación de riesgo de exclusión social. Las personas con discapacidad intelectual es un colectivo que estaría incluido dentro de esta población en situación de riesgo reduciendo su participación en la vida social, aumentando su inseguridad y baja autoestima. Los proyectos de educación en valores permiten generar expectativas positivas en estos colectivos permitiendo a estas personas desarrollarse en un entorno más autónomo. Las actividades de Aprendizaje-Servicio son una herramienta que permite adquirir aprendizajes a través de la experiencia, los alumnos deben planificar, desarrollar y evaluar las actividades destinadas a la mejora de la comunidad.

Con estas experiencias los alumnos de diferentes cursos del Grado en Farmacia y Educación Infantil y Primaria participaron en distintas actividades para mejorar la comunicación entre personas con discapacidad intelectual y los profesionales sanitarios. Igualmente los alumnos consiguieron profundizar más en los contenidos de las asignaturas implicadas y pusieron en práctica sus habilidades comunicativas, además de sensibilizarse con la problemática que rodea a esta población.
\end{abstract}

Palabras clave: inclusión, discapacidad intelectual, Aprendizaje-Servicio, habilidades comunicativas, profesional sanitario.

\title{
1. Introducción
}

Una de las asignaturas pendientes de la educación superior, y probablemente de niveles educativos inferiores, es trabajar en las habilidades comunicativas del estudiante. Las dificultades de comunicación que puede presentar el estudiante pueden repercutir en su futuro desempeño profesional. En el caso de los profesionales sanitarios esta barrera puede limitar su capacidad de compartir y comunicar sus conocimientos a la población general. Un paciente agradece y valora que un buen profesional demuestre eficiencia comunicativa y que sea capaz de transmitirle, de una forma sencilla, todos aquellos aspectos relacionados con su estado de salud. Si nos basamos en que vivimos en un sociedad inclusiva, todas las personas deben de tener las mismas oportunidades y recursos para participar plenamente en la vida económica, social, política y cultural. Aún así siguen existiendo personas vulnerables, que son aquellas que padecen una debilidad (familiar, relacional, socio-económica...) que hace que se encuentren en una 
situación de riesgo de exclusión social. Las personas con discapacidad intelectual y/o del desarrollo es un colectivo que estaría incluido dentro de esta población en situación de riesgo, y que ve en su día a día reducida su participación en la vida social, lo que aumenta su inseguridad y baja autoestima. Las relaciones que se pueden establecer entre futuros profesionales de la salud, como alumnos del Grado en Farmacia, e individuos de estas características están generalmente limitadas a encuentros casuales de la actividad diaria. Estas situaciones no favorecen el desarrollo de las habilidades comunicativas entre ambas poblaciones. Por ello, desde el Grado en Farmacia de la Universidad San Jorge (USJ) se planteó un proyecto de Aprendizaje-Servicio (ApS) llevado a cabo en paralelo en 5 asignaturas diferentes del grado y con colaboraciones puntuales con el Grado en Educación Infantil y Primaria, Mención de Pedagogía Terapéutica. Este proyecto pretendió establecer un vínculo entre ambos colectivos que permita la existencia de una comunicación fluida dentro del ámbito de la salud, eliminando las posibles barreras existentes. Para ello, se contó con la colaboración de la Fundación CEDES (Zaragoza), una entidad sin ánimo de lucro que pretende favorecer la autonomía de las personas con discapacidad intelectual y/o del desarrollo como uno de sus objetivos principales. La finalidad de este proyecto fue favorecer la inclusión social de personas con discapacidad intelectual mejorando sus habilidades de comunicación con los profesionales farmacéuticos y fomentar las competencias comunicativas de los futuros profesionales sanitarios adaptándolas al trato con personas con discapacidad intelectual.

Una metodología ApS propone la participación del alumnado en la vida pública ofreciendo la posibilidad de contribuir, de manera personal o colectiva, en la mejora de algún aspecto de la vida social puede ser de utilidad para nuestros objetivos (Mendia y Moreno, 2010; Martinez, 2010). El ApS permite el desarrollo de competencias, integrando capacidades, habilidades, conocimientos y valores que se movilizan para resolver situaciones reales de manera eficaz (Rubio, 2008). Se trata de un proyecto en el que el alumno se enfrenta a un problema social como es la integración de las personas con discapacidad intelectual en nuestra sociedad fomentando su compromiso y motivación, ya que se les plantea un reto en el que deben implicarse y desempeñar un papel activo. Las actividades organizadas pretenden acercar conceptos distintos sobre el funcionamiento del cuerpo humano y los fármacos a las personas con discapacidad intelectual.

Todas las actividades fueron diseñadas para promover el mayor impacto posible en el aprendizaje del alumno y se sitúan en la zona inferior del cono de aprendizaje de Edgar Dale. Según el mismo, las experiencias que permiten al alumno aumentar su capacidad de retener conocimientos y desarrollar habilidades son la demostración (30\%), grupos de discusión (50\%), prácticas (75\%) y enseñar a otros $(90 \%)$ (Bales, 1996). Asimismo, se fomentan habilidades de pensamiento de orden superior según la Taxonomía de Bloom. Estas experiencias se han desarrollado mediante un trabajo cooperativo, ya que es una de las competencias a las que se hace más referencia en diferentes ámbitos, tanto académico, profesional como social. En el Informe ejecutivo Reflex esta forma de trabajo aparece como una competencia exigida en el ámbito laboral, por lo que debe desarrollarse en el periodo de formación (Barraycoa y Lasaga, 2009). 
Se trata de una metodología de enseñanza-aprendizaje que permite el intercambio y colaboración entre instituciones educativas y sociales. Cada una de las asignaturas vinculadas realizará una actividad de ApS que permita trabajar unos conceptos específicos relacionados con el grado. El conjunto de actividades pretenden que tanto nuestros alumnos como las personas discapacitadas se sientan útiles y protagonistas del cambio, que nuestros alumnos incorporen las herramientas necesarias de comunicación de una forma espontánea, práctica y solidaria y, finalmente, que se produzca un beneficio en la comunidad.

\section{Objetivos}

\subsection{Objetivo general:}

- Mejorar las competencias comunicativas de los futuros profesionales sanitarios adaptándolas al trato con personas con discapacidad intelectual y/o del desarrollo que les permitan familiarizarse con situación habituales en sus salidas profesionales.

\subsection{Objetivos específicos:}

- Sensibilizar a los futuros profesionales farmacéuticos de las limitaciones comunicativas que se pueden encontrar en la sociedad, en este caso con personas discapacitadas intelectualmente.

- Favorecer la comunicación interpersonal entre alumnos y personas discapacitadas fomentando el respeto y la empatía y facilitando el conocimiento del entorno social en el que vivimos.

- Mejorar el grado de autonomía de las personas discapacitadas ofreciéndoles apoyos educativos adaptados y desarrollando su capacidad de razonamiento.

- Potenciar la capacidad de relación social de las personas discapacitadas (expresión de sentimientos, emociones, pensamientos, deseos...) y desarrollar estrategias que ayuden a mejorar el autoconcepto y la autoestima.

\section{Desarrollo de la innovación}

Las actividades de ApS que se diseñaron aparecen descritas a continuación. A modo de resumen los alumnos de $2^{\circ}$ curso en la asignatura de Fisiología Humana I cambiaron su rol y serán "Profesores por un día", diseñando distintas actividades que desarrollaron durante una sesión con alumnos con discapacidad intelectual esta actividad se realizó en colaboración con los alumnos de $4^{\circ}$ del Grado en Educación. Los alumnos de $3^{\circ}$ curso de las asignaturas de Farmacocinética y Farmacología I intentaron responder a las preguntas de “¿Qué hace el fármaco en el cuerpo?” y "¿Qué hace el cuerpo con el fármaco?”, mediante la elaboración de un folleto utilizando un diseño y vocabulario adecuado para este tipo de colectivo. En Biotecnología, asignatura de $4^{\circ}$ curso, tuvieron como objetivo explicar la importancia del DNA mediante el diseño de un vídeo, para lo que utilizaron la herramienta Powtoon. Y para concluir el ciclo, los alumnos de doctorado del grupo de Investigación Greenlife diseñaron una infografía que permitió explicar la importancia de un uso adecuado de los medicamentos para cuidar el medio ambiente. 


\subsection{FISIOLOGÍA HUMANA I: Profesores por un día}

El contenido de esta materia de $2^{\circ}$ curso está centrado en el conocimiento del funcionamiento del cuerpo humano, centrado principalmente en el Sistema Nervioso. Uno de los resultados de aprendizaje que se espera en los alumnos es que adquieran conciencia del carácter global del funcionamiento del organismo humano y de la interacción existente entre los diferentes órganos y sistemas, para posteriormente entender las repercusiones generales sobre la salud que tiene la alteración de cada uno de ellos. Teniendo en cuenta los contenidos de la materia y tras la reunión inicial con los profesores/tutores de CEDES, se determinó que los temas que suscitaban mayor interés para sus alumnos eran los siguientes:

- El gusto.

- El olfato.

- Movimientos: músculo-huesos.

- Dolor.

- Tacto.

Organizados en grupos de 4-5 miembros los alumnos de esta asignatura diseñaron una serie de actividades para desarrollar en el aula que les permitieran explicar uno de estos procesos fisiológicos a personas con discapacidad intelectual. Con la ayuda de los alumnos de la asignatura de Detección e Intervención en centros específicos del Grado en Eduación Infantil y Primaria, prepararon el material didáctico más adecuado para el desarrollo de su tema. Después de una jornada de convivencia en la fundación CEDES, visualización de varios vídeos de divulgación del concepto de sociedad inclusiva y de varios talleres de trabajo cooperativo. Los alumnos plantearon y describieron los objetivos que querían alcanzar y la metodología que iban a seguir para alcanzarlos. Para finalizar la actividad, cada grupo realizó una sesión donde los alumnos de la USJ cambiaron su rol al de profesores y pudieron poner en práctica las actividades diseñadas para pequeños grupos de personas con discapacidad.

Una vez realizada la actividad cada grupo entregó un dossier en el que se incluía una reflexión sobre el proceso de desarrollo de la misma, en ella tenían que justificar la metodología de aprendizaje seleccionada, comentar los vídeos visualización y evaluar la actividad realizada. Esta actividad supuso un $15 \%$ de la calificación final de la asignatura, la evaluación de la misma se realizó mediante rúbricas que estaban centradas en los siguientes aspectos:

- Conocimiento de la materia.

- Capacidad de trabajo en equipo y toma de decisiones.

- Originalidad de las actividades propuestas.

- Capacidad de resolución de problemas.

\subsection{FARMACOCINÉTICA Y FARMACOLOGÍA I: ¿Qué hace el cuerpo con el fármaco?/¿Qué hace el fármaco en el cuerpo?}

La asignatura de Farmacocinética del $3^{\circ}$ Curso del grado de Farmacia se centra en el estudio cinético de los procesos de Liberación, Absorción, Distribución, Metabolismo y Excreción (LADME) de los fármacos, y de sus metabolitos, en los líquidos y tejidos biológicos y su relación con la respuesta farmacológica, terapéutica o tóxica para los seres humanos. Otra de las asignaturas que se imparte de forma paralela y en el mismo curso es la asignatura de Farmacología y Farmacia Clínica I que tiene como objetivo estudiar los efectos de los fármacos sobre los pacientes, los errores de medicación y problemas derivados del uso de medicamentos. Existe una relación evidente entre estas 
asignaturas por ello se decidió que los alumnos realizaran una única actividad que estuvo incluida en los sistemas de evaluación de ambas.

Los alumnos (en grupos de 3) diseñaron un tríptico que les permitió explicar y contestar a estas dos cuestiones: ¿Qué hace el cuerpo con el fármaco? y ¿Qué hace el fármaco al cuerpo? En la primera parte se centraron en explicar qué es la farmacocinética, para qué sirve y qué aplicaciones tiene en el ámbito sanitario. Sin embargo, en la segunda desarrollaron los conceptos asociados a los efectos de los fármacos sobre los seres vivos, objetivo principal de la asignatura de Farmacología I. Todos estos conceptos aparecían explicados en el tríptico de manera clara, sencilla y utilizando el vocabulario y las herramientas más adecuadas para que fueran entendidos por personas con discapacidad intelectual.

Una vez presentada la actividad, los alumnos recibieron la sesión formativa por parte de los tutores de la Fundación CEDES indicándoles a qué tipo de personas iba dirigida la actividad y cómo debían enfocarla. En las siguientes etapas, realizaron el tríptico y se lo presentaron a los profesores, teniendo aquí la primera evaluación de la actividad. A continuación, cada grupo explicó su folleto a los alumnos de la Fundación CEDES. El punto final fue una segunda evaluación de los trabajos por parte de los profesores de CEDES seleccionando aquel que sea más adecuado para sus alumnos. La evaluación de la actividad se realizó mediante una rúbrica y supuso el 10\% de la calificación final de la asignatura. Al tratarse de una actividad desarrollada mediante un único trabajo grupal el sistema de evaluación fue el mismo para ambas asignaturas.

\subsection{BIOTECNOLOGÍA: ¿Qué es el DNA?}

Los alumnos de esta asignatura tuvieron la oportunidad, de forma voluntaria, de elaborar material didáctico que permitió a los alumnos de la fundación CEDES conocer las características del DNA, parte fundamental de los temas introductorios de esta asignatura. Al igual que el resto de los alumnos implicados en el proyecto, aquellos que quisieron participar en esta actividad asistieron a la sesión de formación impartida por los docentes de la fundación CEDES. Con un formato libre, los alumnos de $4^{\circ}$ de Farmacia realizaron una presentación animada utilizando la herramienta informática Powtoon, para fomenter el uso de las Tecnologías de Información y Comunicación (TICs) como herramienta de divulgación científica.

Para finalizar, se organizó una sesión presencial en la que los alumnos de la USJ mostraron los vídeos realizados a los alumnos de CEDES. Por su parte, desde la fundación CEDES se había trabajado previamente este tema para favorecer un intercambio de opiniones en esta sesión.

La evaluación de la actividad se realizó mediante una rúbrica y supuso 1 punto máximo adicional sobre la calificación final de la asignatura. Por su parte, desde la fundación CEDES se decidió realizar también actividades relacionadas con el tema, ya que resultó de especial interés.

\subsection{GREENLIFE: Protegiendo el medio ambiente.}

El grupo de investigación Greenlife de la USJ tiene como una de sus líneas de investigación principales estudiar la toxicidad y ecotoxicidad de diferentes sustancias, como por ejemplo los fármacos. Por ello, a través de esta experiencia se pretendía concienciar de los efectos que los fármacos producen en el medio ambiente y aprender a controlar la contaminación debida a un mal uso de los mismos. Se trató de una actividad de trabajo colaborativo en el que participarán todos los alumnos de doctorado vinculados a este grupo de investigación. La experiencia consistió en la elaboración de una infografía en la que se explicó de manera gráfica y utilizando lenguaje adaptado 
cómo llegan los fármacos al medio ambiente, su efecto nocivo y la importancia de hacer un uso racional de los medicamentos con objeto de prevenir la contaminación medioambiental. Las infografías interactivas se colocaron en las instalaciones de CEDES a la vista de todos los usuarios durante un mes con el objetivo de que pudieran experimentar con ellas, permitiéndoles hacer un seguimiento de todo el proceso. Se evaluó el impacto de la intervención en las personas con discapacidad intelectual mediante un cuestionario realizado con anterioridad a la intervención y tras el mes de exposición de las infografías.

\section{Resultados}

El desarrollo de todas estas experiencias permitió a los alumnos mejorar sus calificaciones en el apartado de trabajo en grupo del sistema de evaluación de cada asignatura con respecto a años anteriores. Y por otro lado, ha permitido a los alumnos desarrollar y alcanzar competencias a menudo olvidadas como compromiso, responsabilidad y participación en la comunidad.

El 40\% de los alumnos del Grado en Farmacia no había estado en contacto con personas con discapacidad intelectual y/o del desarrollo hasta el momento, según una encuesta que se les realizó al principio del proyecto. Una de las primeras reflexiones que los profesores teníamos interés en conocer era qué habían sentido los alumnos al conocer la realización de estas actividades. En la Figura 1 aparecen representadas las respuestas dadas por ellos mediante una nube de palabras, apareciendo representadas con mayor tamaño aquellas más repetidas.

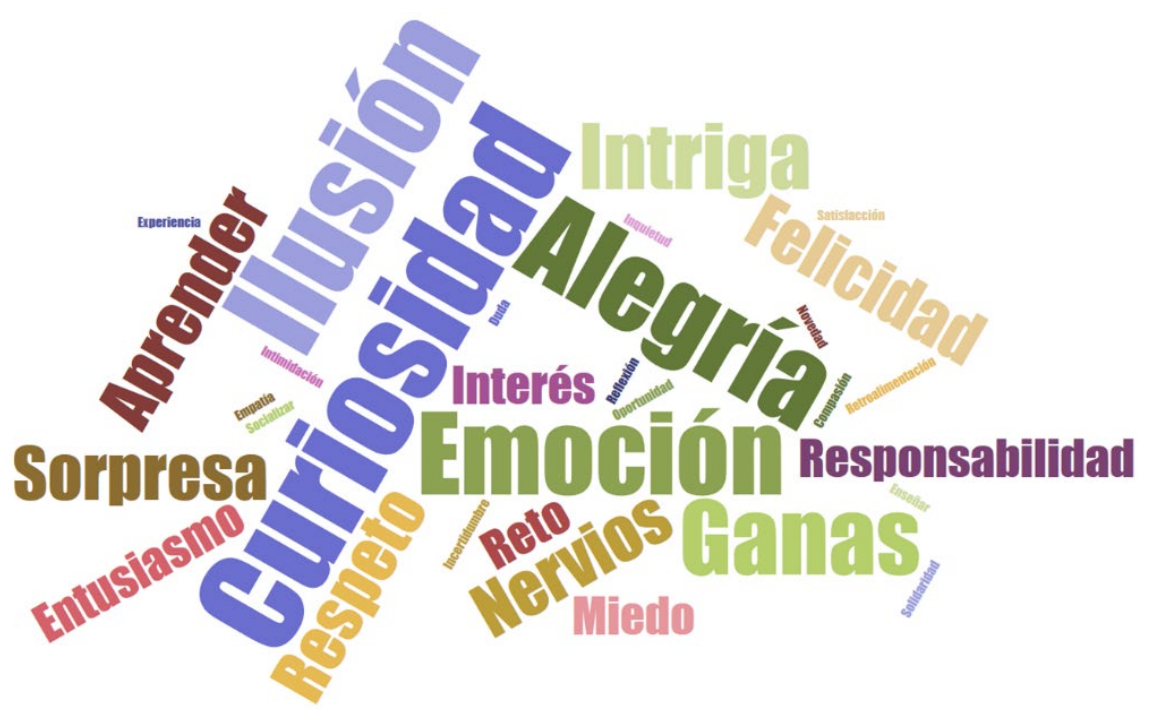

Figura 1. Definición en tres palabras de lo que sintieron los alumnos al conocer que iban a realizar dicha actividad (https://www.jasondavies.com/wordcloud/).

Todos los materiales diseñados por los alumnos durante esta experiencia (Figura 2) fueron cedidos a la fundación CEDES con el objetivo de que pudieran 
trabajar sobre estos temas con más profundidad y al ritmo marcado por cada uno de sus usuarios.
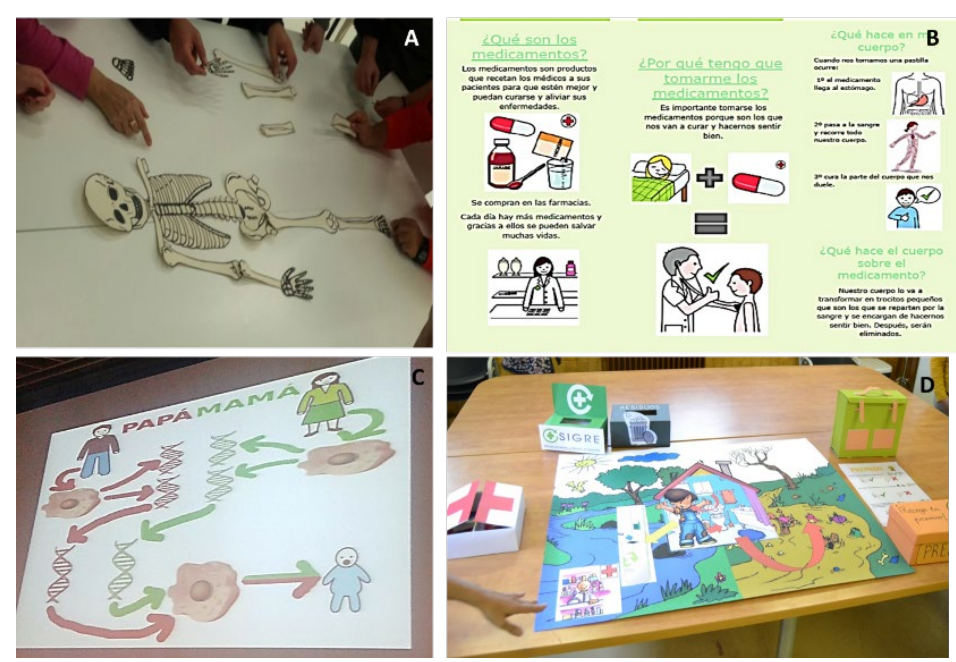

Figura 2. Ejemplo de alguno de los materiales diseñados por los alumnos en cada una de las asignaturas: A) Fisiología Humana I; B) Farmacología I y Farmacocinética; C) Biotecnologías y D) Alumnos de Doctorado en Medio ambiente.

Cabe destacar que en el caso de la actividad sobre el DNA, los alumnos de CEDES habían preparado previamente el tema lo que hizo que la realización de la actividad fuera muy dinámica y que adquirieran unos conocimientos básicos del DNA que les resultaron nuevos y de su interés.

Obviamente también era importante conocer la opinion de los profesionales de la fundación CEDES sobre el desarrollo de estas actividades. Por ello se les pidió que completarán un cuestionario después de realizar las distintas actividades. Los resultados cuantitativos del mismo aparecen reflejados en la Figura 3. 


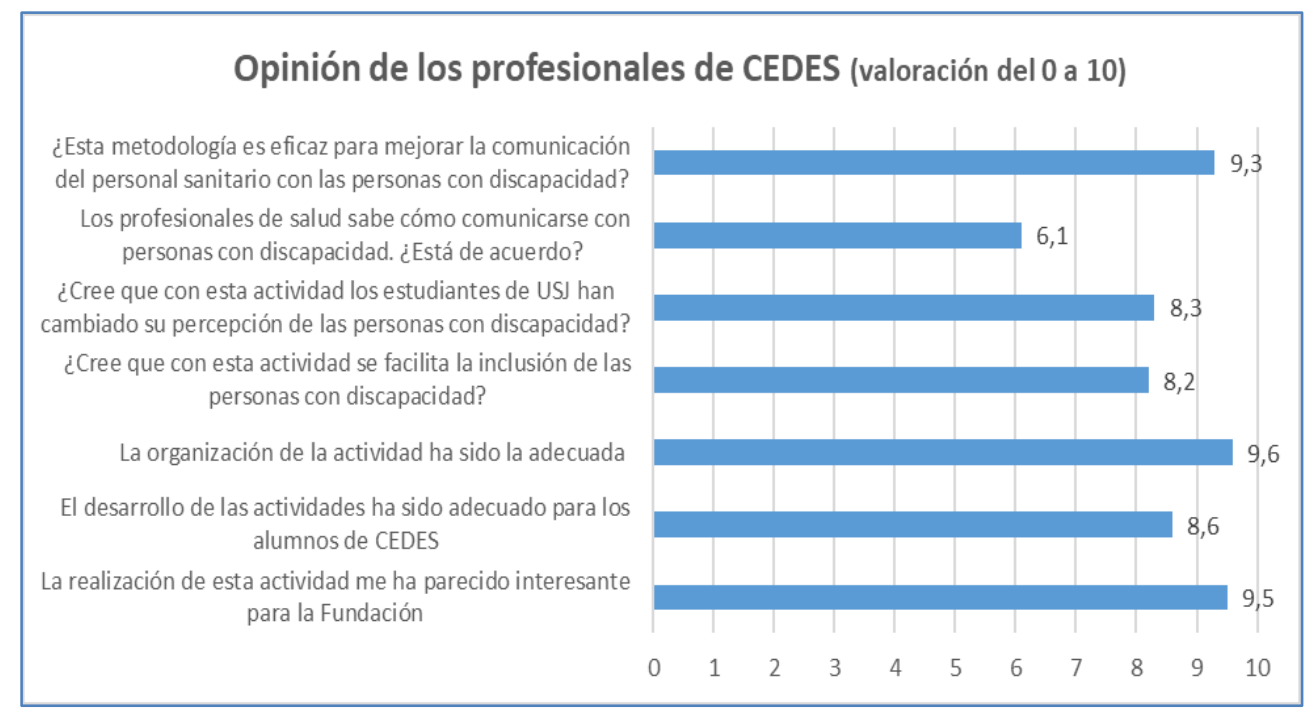

Figura 3. Resultado cuantitativo de la opinión de los profesionales de CEDES ( $n=8)$ sobre las actividades realizadas.

Dadas las características de la población con la que se trabaja resulta complicado evaluar la idoneidad solo mediante información cuantitativa, por ello, se decidió realizar también cuestiones abiertas para evaluar otros aspectos de forma cualitativa. Los docentes de las asignaturas consideraron oportuno realizar este tipo de preguntas ya que podían ser un reflejo más real del impacto de la actividad. Una de las preguntas que mejor representaba dicho impacto aparece reflejada en la Figura 4.

\section{¿Qué capacidades/habilidades se han trabajado/han mejorado en los alumnos de USJ con esta actividad?}

- Contacto con personas con discapacidad.

- Conocer posibles formas de comunicarse.

- Acceder a las personas con dificultades.

- Integración social.

- Ver otra percepción del ámbito de la discapacidad.

- Respetar ritmos individuales.

- Han adaptado contenidos complejos a un nivel de comunicación al que no están acostumbrados. Han usado pictogramas y ejemplos reales.

- Con este tipo de actividades se hacen visibles las personas con discapacidad.

- Se es consciente de que todos podemos aprender.

- Se conocen sus capacidades y dejamos a un lado las limitaciones.

Figura 4. Resultados de la valoración cualitativa por parte de los profesionales de CEDES $(n=8)$.

En la Figura 5 aparecen representadas las opiniones cuantitativas de los alumnos de la USJ sobre las distintas actividades. 


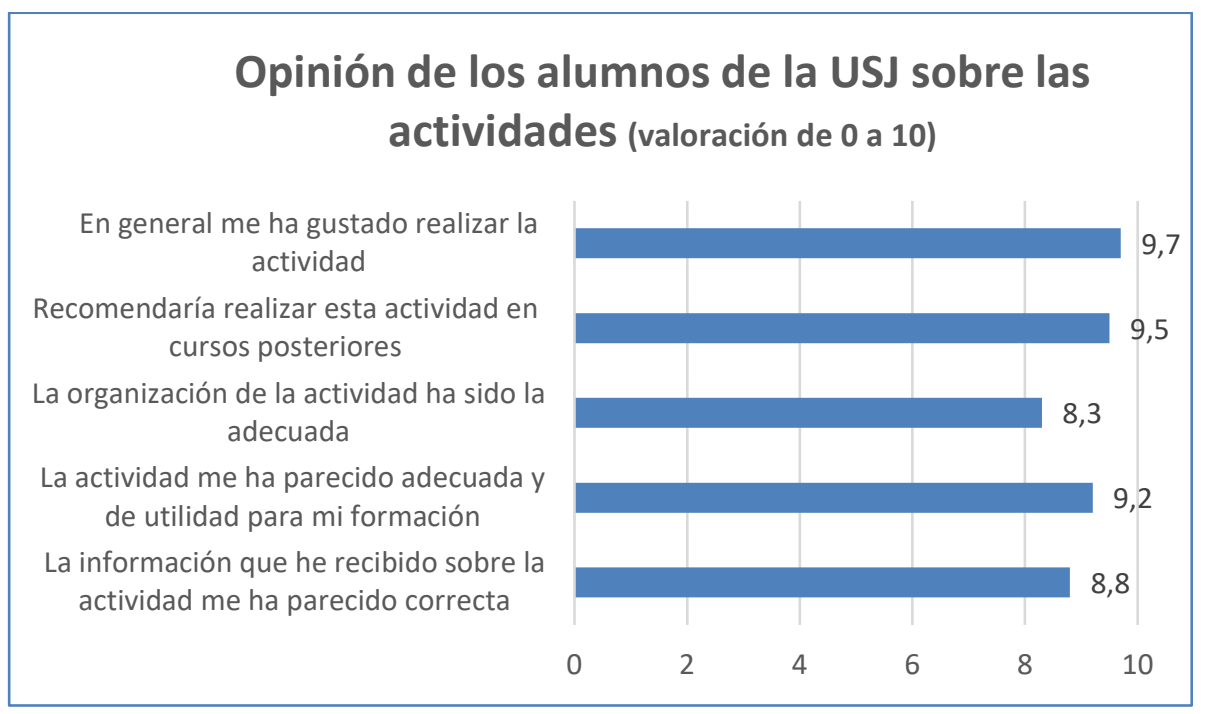

Figura 5. Resultados de la valoración de las distintas actividades realizadas por los alumnos de la USJ (n=71, incluidos los alumnos de las 5 asignaturas).

Al igual que en el caso de los profesionales de CEDES también se realizaron varias preguntas abiertas sobre las actividades. En la mayoría de los casos las respuestas otorgadas por los alumnos permitieron confirmar que se habían logrado los objetivos propuestos con esta experiencia como aparece reflejado en la Figura 5.

\section{Indica qué crees que te ha aportado esta actividad}

- En general ha sido una experiencia bonita que repetiría. Me ha ayudado a ver a estas personas desde otro punto de vista.

- Conocimientos sobre la gente con discapacidad intelectual ya que nunca había tratado. Ha sido una experiencia muy positiva.

- Conocer la realidad de estas personas y romper barreras y perjuicios.

- Me ha acercado a una realidad que no conocía.

- Me ha aportado la capacidad de enseñar a alguien algo que no conocían.

- Gratificación personal.

- Me ha aportado una experiencia tanto en el ámbito profesional ya que hemos organizado una clase y en el social.

- Aprender a valorar las capacidades de las personas con discapacidad que antes pensaba que no tenían.

- Me ha ayudado a que si en un futuro tengo que hacerlo no tenga tanta inseguridad.

- Ser capaz de simplificar una serie de conocimientos para adaptarlos a personas con un nivel de comprensión bajo.

- Es una experiencia personal muy reconfortante.

- Me ha permitido relacionarme con personas con discapacidad.

Figura 5. Opiniones de los alumnos sobre lo que les ha aportado la realización de esta actividad (encuesta realizada a 71 alumnos, sólo aparecen las respuestas más repetidas). 
Con respecto a la opinión de los profesores de la USJ, 6 docentes en total, todos los participantes estaban de acuerdo en la satisfacción que supone este tipo de actividades a nivel docente. Al finalizar todas las actividades se realizó un focus group donde todos los docentes implicados intercambiaron sus opiniones e impresiones generales sobre el proyecto y sobre su actividad en particular. Ver disfrutar a los usuarios de CEDES y ser partícipes de su inclusión en la sociedad es uno de los aspectos más remarcado por todos.

\section{Conclusiones}

Para todas las asignaturas que han participado en este proyecto, tanto del Grado en Farmacia como del Grado en Educación, ha supuesto un reto enfrentarse a una situación real con personas con discapacidad intelectual y/o del desarrollo. Todos los alumnos de la USJ tuvieron la oportunidad de desarrollar habilidades comunicativas además de profundizar en los conceptos teóricos básicos de las diferentes asignaturas. Por otro lado, la multidisciplinaridad de esta experiencia ha permitido a los profesores que han participado en ella colaborar con otros docentes y compartir experiencias entre ellos, hecho que ha repercutido sobre el entusiasmo docente de todos ellos.

El uso de una metodología de enseñanza-aprendizaje como el ApS ha permitido subrayar el valor de las actividades educativas solidarias al servicio de la comunidad y desarrolla su potencial formador conectándolas con el aprendizaje formal. Se trata de un aprendizaje basado en la exploración, la acción y la reflexión. Se educa a los alumnos a través de valores y para la ciudadanía que permite adquirir no solo competencias educativas sino también competencias para la vida. Esta herramienta permite un aprendizaje sistematizado, asociado a un servicio o una carencia de la comunidad, y tiene que permitir evaluar lo que sus protagonistas aprenderán antes, durante y después de su realización. Esta actuación se basa en la convicción de que una de las formas más efectiva de aprendizaje es enseñando a otros, ya que para ello el alumno debe dominar la materia y ser capaz de enfocarlo de distintas formas para que cualquier individuo sea capaz de entenderlo.

El uso de las herramientas TIC es otro de los beneficios que obtienen los alumnos ya que serán básicos para llevar a cabo las actividades propuestas. Esta experiencia ha supuesto una colaboración de docentes de varias asignaturas del Grado en Farmacia (de $2^{\circ}$ a $5^{\circ}$ curso/doctorado) y los del Grado en Educación en el que se ha guiado a los alumnos a través de la realización de los distintos tipos de trabajos como la planificación de una clase docente, elaboración de un tríptico informativo o diseño de un vídeo explicativo y una infografía. En todos ellos el alumno es el protagonista de su propio proceso de aprendizaje.

La elección de una metodología mediante ApS mejora el servicio a la comunidad mientras que el servicio a la comunidad mejora el aprendizaje, la motivación y le da más sentido.

\section{Referencias}

BALES, E (1996). "Corporate Universities vs Traditional Universities: Friends or Foes? Third Annual EDINEB (Educational Innovations in Economics and Business) International Conference, Orlando (USA). 
BARRAYCOA Y LASAGA (2009). "Competencias e inserción laboral: un análisis de la empleabilidad en los recién licenciados en Ade y Economía”. Madrid: CEU ediciones.

INFORME EJECUTIVO REFLEX (2007). "El profesional flexible en la Sociedad del Conocimiento". Madrid, ANECA.

MARTÍNEZ M (2010). Aprendizaje servicio y responsabilidad social de las Universidades. Barcelona, Ed. Octaedro.

MENDIA R, MORENO V (2010). Guía zerbikas 3: Aprendizaje y servicio solidario, aprender a emprender sirviendo a la comunidad. Bilbao. Zerbikas Fundazioa.

RUBIO L (2008). Guía zerbikas 0: Aprendizaje y servicio solidario. Bilbao, Zerbikas Fundazioa. 